\title{
Estimativa do número básico de reprodução R0 do COVID-19 nos países da América do Sul
}

\author{
Estimate of the basic number of reproduction RO of COVID-19 in South American countries
}

Carlos Dutra ${ }^{1}$

\section{RESUMO}

No presente estudo foi realizada a estimativa do número básico de reprodução R0 do COVID-19 nos 12 países da América do Sul utilizando método empírico baseado na taxa de crescimento do número de casos novos no início da fase exponencial da epidemia. Foram encontrados os seguintes valores de R0: 1,94 - Argentina, 2,18 - Bolívia, 3,07 - Brasil, 2,50 - Chile, 1,94 - Colômbia, 3,42 - Equador, 1,01 - Guiana, 2,06 - Paraguai, 1,89 - Peru, -0,13 - Suriname, 1,13 - Uruguai, 1,85 - Venezuela. A partir desses valores de R0 estimados tem-se que o COVID-19 tem a natureza epidêmica (R0>1) em quase todos países da América do Sul a exceção do Suriname com valor de $\mathrm{R} 0<1$. Destaca-se a importância da realização dos testes de diagnóstico COVID-19 de foma mais sistemática para um maior conhecimento da evolução da doença nos países auxiliando nas tomadas de decisões de combate ao COVID-19 por parte do s órgâos governamentais e da sociedade como um todo.

Palavras-chave: R0; coronavirus; covid-19; número básico de reprodução

\section{ABSTRACT}

In the present study, the basic number of reproduction RO of COVID-19 was estimated in the 12 countries of South America using an empirical method based on the growth rate of the number of new cases at the beginning of the exponential phase of the epidemic. The following values of Ro were found: 1.94 - Argentina, 2.18 - Bolivia, 3.07 - Brazil, 2.50 - Chile, 1.94 - Colombia, 3.42 - Ecuador, 1.01 - Guyana, 2.06 - Paraguay, 1.89 - Peru, -0.13 - Suriname, 1.13 Uruguay, 1.85 - Venezuela. From these estimated R0 values, COVID-19 has an epidemic nature (R0>1) in almost all South American countries except Suriname with a value of $\mathrm{R} 0<1$. We highlight the importance of conducting the covid-19 diagnostic tests of more systematic foma for a greater knowledge of the evolution of the disease in the countries assisting in the decision-making of the fight against COVID-19 by the government and society as a whole.

Keywords: R0; coronavirus; covid-19; basic number of reproduction

Como citar este artigo / How to cite this article

Dutra C. Estimativa do número básico de reprodução R0 do COVID-19 nos países da América do Sul Estimate of the basic number of reproduction R0 of COVID-19 in South American countries. InterAm J Med Health 2020;3:e202003035. 


\section{INTRODUÇÃO}

A COVID-19 doença causada pelo virus SARS-CoV2 surgiu na cidade de Wuhan, na Provincia de Hubei (China) em Dezembro de 2019; e em 11 de março de 2020 ja foi declara como Pandemia pela Organizaça de 2020 já foi declara como Pandemia pla Organizaçao Mundial da Saúde - OMS, nessa data contava com 80955 infectados na China e 37364 infectados fora da China (WHO SR1,2020 e WHO SR-51, 2020). Em 01 de junho de 2020 registrou-se 6.057 .853 casos (122.917 nas últimas $24 \mathrm{~h}$ ) com um total de 371.166 mortes (4.000 nas últimas 24h) conforme OMS (WHO SR-133,2020); dados referentes aos países da América do Sul estão na Tabela 1.

O número básico de reprodução RO de uma doença constitui-se num dos parâmetros essenciais para dimensionar a intensidade de transmissão de uma doença infecciosa. O RO pode ser definido como o número médio de infecções secundárias produzidas quando um indivíduo infectado é introduzido na população; quando RO é maior que um o surto pode transformar-se em uma epidemia, e quando RO menor que um o surto torna-se extinto (Anderss vários trabathos tem sido reatzados no sentido de sua determinaca, Liu et al. [1] compilou estinativas de RO 01 janeiro a 7 de fevereiro 2020 com um na China de 01 janeiro a 7 de fevereiro 2020 com um total de 13 trabalhos encontrando uma estimativa média de 3,28 para o R0 da COVID-19. Alimohamadi et al. [2] realizou um levantamento sistemático sobre valores de RO para a China e encontrou 3,32 considerando 23 trabalhos anteriores. Rahman et al. [3] realizaram uma revisão de literatura encontrando 21 métodos diferentes de determinação do RO em um total de 103 trabalhos, entre janeiro e março 2020, com estimativas de R0 para China (em especial valor médio de 2,71 para Wuhan) e diversas regiões bem como para outros países como Coréia do Sul, Iran, Itália, Argéria, Israel. Estes autores destacam ainda trabalhos que indicaram os impactos do distanciamento social no R0 com redução nos valores de R0 na China de 2,92 para 1,40 e na Itália de 2,38 para 1,66. O varor de RO pode depender do método utilizado, bem como a série temporal de dados de casos infectados que é utilizada.

O presente trabalho visa determinar valores de R0 para o início da fase exponencial de crescimento do número de novos infectados nos países da América do Sul.

MÉTODO

De acordo com Wallinga \& Lipsitch [4] a primeira informação que se tem de uma epidemia é o número de casos que aumentam diariamente, na fase inicial essas contagens aumentam de forma exponencial. A taxa de crescimento exponencial, $r$ mede a variação do número de casos de novos infectados com o tempo e está relacionada com o valor do número reprodutivo básico RO pela equação:

$R O=1+r$. Tc $(1)$

Esta equação é uma aproximação válida para a fase inicial do crescimento exponencial da epidemia. 0 termo Tc é denominado intervalo serial que representa a média da duração de tempo entre um infectante primário com início dos sintomas e um infectado secundário com início dos sintomas.

O intervalo serial do COVID-19 tem sido estimado através da análise de documentação de registros clínicos de pacientes que requer a investigação para determinar os pares infectado-infectantes, obtendo uma distribuição de valores de intervalos seriais aos quais é ajustada distribuições matemáticas tais como Gama e Weibull. Aghaali et al. [5] determinou o intervalo serial para Qom ne 4,55 dis e desino padro de 3,3 das. Wang édia de 4,55 dias e desio padrao de 3,3 dias. Wang et al. 6] examinando 27 registros de cadela de transmissão encontrou para Shenzhen na China um intervalo serial que seguia uma distribuição de Weibull com média 5,9 dias e desvio padrão de 4,8 dias. Nishiura et al. [7] obteve uma distribuição LogNormal com média de 4,7 dias e desvio padrão de 2.9 dias para o intervalo serial com casos do Vietnã, Coréia do Sul, Alemanha, Taiwan, China e Singapura. You et al. [8] encontrou para 6 províncias Chinesas encontrou uma média de 4,6 dias e um desvio padrão de 5,55 dias para o intervalo serial.

Considerando que a taxa de crescimento exponencial, , pode ser obtida do ajuste de mínimos quadrados de uma função exponencial (IIt)=10 x ert) aos dados de número de casos de COVID-19, e adotando um valor para Tc, podese estimar o valor de Ro no início da fase exponencial da epidemia nos diferentes países.

\section{APLICAÇÃO E RESULTADOS}

Para a implementação do método, foram extraídos dados de incidência ( ou seja Casos Novos) da Epidemia COVID-19 nos países da América do Sul no repositório de dados "Data on COVID-19 (coronavirus) by Our World in Data" disponiveis no site: https//github.com/owid/coviddisponiveis no site: https.//github.com/owid/c novos de cada país foi aplicada a "suavização" média das vezes os 10 primeiros valores de números de casos móvel com intervalo de 7 dias. Esta "suavização" é novos "suavizados" pela média móvel. Na Figura 1 temnecessária pois os casos novos apresentam com intervalo se a representação gráfica do ajuste exponencial na fase apenas de um dia uma ocorrência irregular, então faz-se inicial do crescimento de casos novos no Brasil. Na Tabela 1 necessário obter-se uma média ao longo de um tempo são apresentados os resultados do ajuste de determinação maior para tornar evidente o comportamento exponencial da taxa de crescimento $r$. Conhecendo-se os valores de $r$ do crescimento do número de casos novos. Após foi e adotando a determinação de Nishiura et al. [7] de 4,7 \pm utilizado o método de regressão não linear de mínimos 2,9 para o intervalo serial Tc, foi possível determinar o RO quadrados de função exponencial ao conjunto de valores através da Equação 1 cujos resultados estão na Tabela 1. que representam o início da fase exponencial, na maioria

Figura 1 - Ajuste de função exponencial aos dados de Casos Novos do Brasil "suavizados" com média móvel.

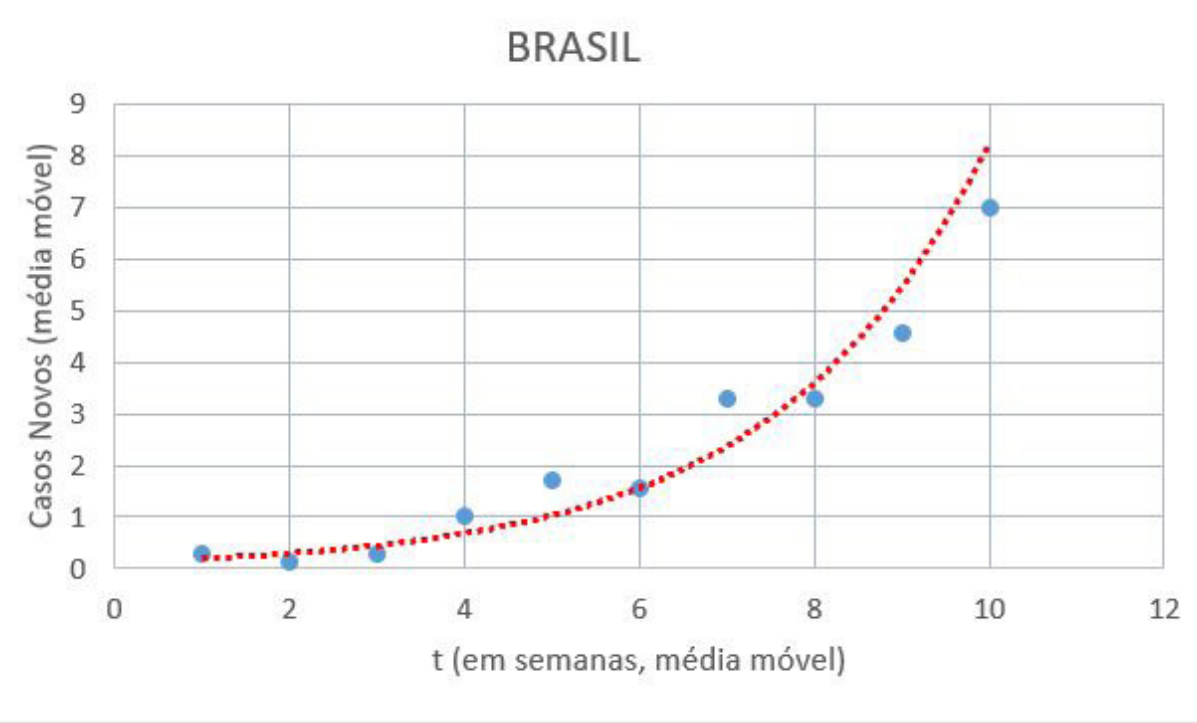

Tabela 1 - Dados COVID-19 dos países da América do Sul e resultados da aplicação do método.

\begin{tabular}{|l|c|c|c|c|c|}
\hline & $\mathbf{N}^{\circ}$ Casos Confirmados & $\mathbf{N}^{\circ}$ Mortes & $\mathbf{N}^{\circ}$ Casos Novos & \multicolumn{2}{c|}{ Resultados } \\
\hline \multicolumn{1}{|c|}{ P1 de junho 2020 } & \multicolumn{3}{|c|}{} & r & R0 \\
\hline Argentina & 16214 & 1512 & 530 & 0,201 & 1,94 \\
\hline Bolivia & 9592 & 861 & 310 & 0,251 & 2,18 \\
\hline Brasil & 498440 & 33274 & 28834 & 0,441 & 3,07 \\
\hline Chile & 99688 & 4830 & 1054 & 0,320 & 2,50 \\
\hline Colômbia & 28236 & 1548 & 890 & 0,199 & 1,94 \\
\hline Equador & 39098 & 527 & 3358 & 0,514 & 3,42 \\
\hline Guiana & 152 & 2 & 12 & 0,002 & 1,01 \\
\hline Paraguai & 986 & 22 & 11 & 0,226 & 2,06 \\
\hline Peru & 155671 & 7386 & 4371 & 0,190 & 1,89 \\
\hline Suriname & 14 & 2 & 1 & $-0,241$ & $-0,13$ \\
\hline Uruguai & 821 & 5 & 22 & 0,028 & 1,13 \\
\hline Venezuela & 1459 & 89 & 14 & 0,181 & 1,85 \\
\hline
\end{tabular}

Vonte: Dados COVID-19 obtidos relatórios WHO (SR133, 2020) e resultados do autor. 


\section{CONSIDERAÇÕES FINAIS}

O número básico de reprodução RO constitui-se em um dos parâmetros fundamentais da Epidemiologia porque o surto de uma doença só se caracteriza como epidemia quando o Ro é maior que 1; caso contrário quando o R0 é menor que 1 ocorre o declínio do número de infectados. Nesse estudo verificou-se que quase todos os países da América do Sul tiveram um RO no início do contágio da COVID-19 com RO > 1 caracterizando a doença como epidemia; somente no Suriname ocorreu uma variação negativa do número de casos novos com o tempo resultando em um $\mathrm{RO}<1$ com pouquissimos casos onde muitos deles devem ser casos importados cuja transmissão em maior escala foi evitada provavelmente com o devido isolamento dos infectados antes de iniciar a transmissão comunitária. Dos países com R0>1 destaca-se em primeiro lugar o Equador com $\mathrm{R} 0=3,42$ e em segundo lugar o Brasil com $\mathrm{RO}=3,07$ seus 39098 infectados representam 2219 por 1 milhão de habitantes, e o Brasil com seus 498440 infectados representam 2346 por 1 milhão de habitantes (dados de prevalência do site "Worldometer" - https://www.worldometers. info/coronavirus/). Chama a atenção os dados do Peru, embora o R0 inicial seja de 1,89 similar ao da Colômbia e Argentina, o país apresenta o segundo maior número de casos infectados da América do Sul considerando os dados, entretanto o Peru tem $5 x$ mais testes realizados que a Colômbia e quase 10x mais testes realizados do que a Argentina conforme os dados do site "Worldometer". O que nos pemite colocar mais uma questão importante que é a realização dos testes de diagnóstico do COVID-19, pois somente através deles poderemos ter dados como RO ou a variação do R0 com o tempo, o número de reprodução efetivo $\mathrm{R}(\mathrm{t})$ que nos permitirá monitorar o avanço da COVID-19 podendo utilizar a sua estatística de forma proativa como ferramenta para a tomada de decisão por parte dos governos e diversos setores da economia e da saúde para reduzir o impacto desta doença na sociedade enquanto não se descobre uma vacina.

\section{REFERÊNCIAS}

1. Anderson, R. May, R. Infectious Diseases of Humans. 1991. Oxford: Oxford University Press.
2. Liu, Y.; Gayle, A.; Wilder-Smith, A., Rocklov, J. The reproductive number of COVID-19 is higher compared to SARS coronavirus. Journal of Travel Medicine. 2020, vol 27 n.2 Pg. 1-4 https://doi.org/10.1093/jtm/taaa021

3. Alimohamadi, Y., Taghdir, M., Sepandi, M. Estimate of the Basic Reproduction number for COVID-19: A Systematic Review and Meta-analysis. J.Prev.Med> Public Health 2020, 53, 151-157 doi: https://doi.org/10.3961/jpmph.20.076

4. Rhaman, B.; Sadraddin, E.; Porreca, A. The basic reproduction number of SARS-CoV-2 in Wuhan is about to die out, how abour the rest of the World? Reviews in Medical Virology. 2020 https://onlinelibrary.wiley.com/doi/full/10.1002/rmv.2111

5. Wallinga, J.; Lipsitch, M. How generation intervals shape the relationship between growth rates and reproductive numbers. Proc. R. Soc. B. 2007, n. $274,599-604$ doi:10.1098/rspb.2006.3754

6. Aghaali, M.; Kolifarhood, G.; Nikbakht, R.; Saadati, H.; Nazari, S. Estimation of the Serial Interval and basic reproduction number of COVID-19 in Qom, Iran, and Three Other Countries: A data-driven analysis in the early phase of the outbreak. 2020. PMID: 32473049 doi: 10.1111/tbed.13656

7. Wang. K.; Zhao, S.; Liao, Y.; Zhao, T.; Wang, X.; Zhang, X.; Jiao, H.; Li, H.; Wang, M.; Xiao, L.; Wang, L.; He, D. Estimating the Serial Interval of the Novel Coronavirus Disease (COVID-19) Based on the Public Surveillance data in Shenzhen, China From January 19 to February 22, 2020. PMID: 324552648 doi: https://doi.org/10.1111/tbed.13647

8. Nishiura, H.; Linton, N.; Akhmetzhanov, A. Serial interval of novel coronavirus (COVID-19) infections. International Journal of Infectious Diseases v. 93, 2020, 284-286 https://doi.org/10.1016/j.ijid.2020.02.060

9. You, C.; Deng, Y.; Hu, W.; Sun, J.; Lin, Q.; Zhou, F.; Pang, C.H.; Zhang, Y.; Chen, Z.; Zhou, X. Estimation of the time-varying reproduction number of COVID-19 outbreak in China (2/17/2020). 2020. Int J Hyg Environ Health. 2020. http://dx.doi.org/10.2139/ssrn.3539694 de habilidades e capacidades motoras: uma revisão sistemática. Revista Paulista de Pediatria, 35(4), 464-471. 